\title{
Measurement of the Temperature- Dependent Recombination Lifetimes in Photovoltaic Materials
}

S.W. Johnston

Colorado School of Mines, Golden, CO

R.K. Ahrenkiel

National Renewable Energy Laboratory

Presented at the National Center for

Photovoltaics Program Review Meeting

Denver, Colorado

September 8-11, 1998

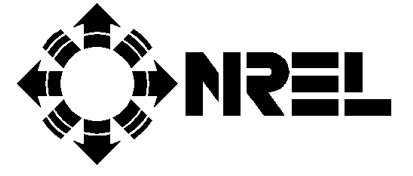

National Renewable Energy Laboratory 1617 Cole Boulevard Golden, Colorado 80401-3393

A national laboratory of the U.S. Department of Energy Managed by Midwest Research Institute for the U.S. Department of Energy under contract No. DE-AC36-83CH10093

Work performed under task number PV903101

October 1998 


\begin{abstract}
NOTICE
This report was prepared as an account of work sponsored by an agency of the United States government. Neither the United States government nor any agency thereof, nor any of their employees, makes any warranty, express or implied, or assumes any legal liability or responsibility for the accuracy, completeness, or usefulness of any information, apparatus, product, or process disclosed, or represents that its use would not infringe privately owned rights. Reference herein to any specific commercial product, process, or service by trade name, trademark, manufacturer, or otherwise does not necessarily constitute or imply its endorsement, recommendation, or favoring by the United States government or any agency thereof. The views and opinions of authors expressed herein do not necessarily state or reflect those of the United States government or any agency thereof.
\end{abstract}

Available to DOE and DOE contractors from:

Office of Scientific and Technical Information (OSTI)

P.O. Box 62

Oak Ridge, TN 37831

Prices available by calling 423-576-8401

Available to the public from:

National Technical Information Service (NTIS)

U.S. Department of Commerce

5285 Port Royal Road

Springfield, VA 22161

703-605-6000 or 800-553-6847

or

DOE Information Bridge

http://www.doe.gov/bridge/home.html 


\title{
Measurement of the Temperature- Dependent Recombination Lifetimes in Photovoltaic Materials
}

\author{
S. W. Johnston* and R. K. Ahrenkiel \\ National Renewable Energy Laboratory, Golden, CO 80401 \\ *Materials Science Program, Colorado School of Mines, Golden, CO 80401
}

\begin{abstract}
Lifetime spectroscopy is a valuable tool for the characterization of photovoltaic materials. Measured lifetime values are inherently dependent on the defect and impurity densities present in the material. Injection-level and temperature dependencies of the recombination rate further characterize the material and possibly provide information for the identification of specific impurities. Also, trapping levels may be determined by observing their temperature-dependent thermal emission. Measured examples include surface-passivated, float-zone silicon and highquality, undoped GaAs. Excess-carrier-decay curves are recorded from 80 to $300 \mathrm{~K}$ using a lifetime-measurement technique called ultrahigh frequency photoconductive decay.
\end{abstract}

\section{INTRODUCTION}

The minority-carrier lifetime is an important semiconductor device parameter. It is sensitive to impurities and defects that are present in the material (1); thus, its value correlates to material quality. In the case of photovoltaic devices, lifetime relates to efficiency. While a lifetime value can give an overall quality rating and defect concentration $(2,3)$, specific characteristics of the carrier decay can provide additional information. The lifetime values at various levels of excess-carrier density can provide ratios of minority- to majority-carrier capture cross sections and be useful for identifying specific impurities (4). The temperature dependence of carrier recombination further characterizes the material. First, capture-crosssection temperature dependency can assist in identification of impurities (5-10). Second, trapping can be determined by temperature-dependent thermal emission of carriers. Finally, lifetime can be measured at operating temperature for low-temperature devices.

\section{EXPERIMENT}

The lifetime is measured using a contactless, transient-response technique known as ultrahigh frequency photoconductive decay (UHFPCD) (11-14). The sample is inductively coupled to a small, three-turn, loop antenna that is $5 \mathrm{~mm}$ in diameter and driven by an approximately $500-\mathrm{MHz}$ sinusoidal source. The antenna can be tuned to resonance by adjusting a variable capacitor, frequency, and/or mutual coupling to the sample. Without a light source for creating excess carriers, the circuit's zero signal corresponds to the sample's 
dark conductivity. Excess carriers are created using a 355-nm pumped optical parametric oscillator that outputs laser light pulses of tunable wavelengths from ultraviolet to infrared. For each laser pulse, changes in sample conductivity disrupt the circuit balance and give a proportional voltage signal that is detected and recorded using an oscilloscope. The carrier's mobility changes with temperature, and this affects the initial conductivity value, which is represented as the initial signal value in units of volts. Mobility may also change with carrier concentration due to increased carrier-carrier scattering at higher carrier densities. Excess carrier values and the correlating carrier-carrier scattering are assumed to be small and are neglected in the lifetime calculation.

To make temperature-dependent measurements, the sample is placed in a vacuuminsulated Dewar, cooled using liquid nitrogen to $78 \mathrm{~K}$, and allowed to warm to room temperature. The sensing coil is mounted at the end of semirigid coaxial cable and embedded in electrically insulating plexiglass for mounting the sample. A type-T thermocouple wire runs along the coaxial cable, and the exposed junction is placed in contact with the sample. Allowing the sample to warm from $78 \mathrm{~K}$ to room temperature takes approximately 2 hours, but this time can be reduced by using heat-supplying resistors surrounding the sample mount. Each captured lifetime measurement averages the decay curve for a few seconds, and the sample remains at the recorded average temperature within a degree or two.

Data are presented from measurements of Si and GaAs. The Si sample is a cleaved piece from an n-type, float-zone wafer. The laser wavelength is tuned to $1064 \mathrm{~nm}$, which is absorbed in silicon with absorption coefficients of $\alpha=10 \mathrm{~cm}^{-1}$ at $300 \mathrm{~K}$ and $\alpha=0.2 \mathrm{~cm}^{-1}$ at 77 $\mathrm{K}$. This weakly absorbed light promotes excess-carrier excitation throughout the thickness of the sample. The surfaces are passivated with a grown oxide that reduces the surface recombination velocity by more than six orders of magnitude (15). The GaAs sample was grown undoped, although a residual doping density of less than $10^{15} \mathrm{~cm}^{-3}$ does exist and characterizes the 2- $\mu \mathrm{m}$ layer as n-type. The samples are grown in a double heterojunction structure where $300 \AA \mathrm{Al}_{0.6} \mathrm{Ga}_{0.4} \mathrm{As}$ layers provide surface passivation. The GaAs sample was grown under lower temperature processing conditions for more optimum undoped GaAs. A laser wavelength of $670 \mathrm{~nm}$ is used to create excess carriers in the GaAs.

\section{RESULTS}

The Si sample's excess-carrier-decay curves for the series of temperatures between $78 \mathrm{~K}$ and room temperature are plotted in Fig. 1 . At $78 \mathrm{~K}$, the decay rate corresponds to a minoritycarrier lifetime of $20 \mu \mathrm{S}$. As the temperature increases, the decay rate decreases and corresponding lifetimes are larger. The low-injection lifetime at room temperature is $100 \mu \mathrm{S}$. A high-injection regime becomes more defined. The ratio of high-injection to low-injection lifetimes relates to the capture cross sections for both carrier types. This ratio can help identify the dominant defects controlling recombination. Temperature dependence can also provide information about capture cross sections and possibly identify the impurities or defects present in the material.

In order for the lifetime to increase with increasing temperature, the capture cross section must correspondingly decrease. For Coulomb capture, the capture cross section is calculated to vary at $\mathrm{T}^{-\mathrm{n}}$, with $\mathrm{n}$ being as large as 2 or $4(16,17)$. Polarizability of a neutral center can also account for a cross section that increases with decreasing temperature (17). These parameters for capture cross section are included in a recombination model incorporating Shockley-ReadHall (SRH) recombination given in Eq. $1(18,19)$. 


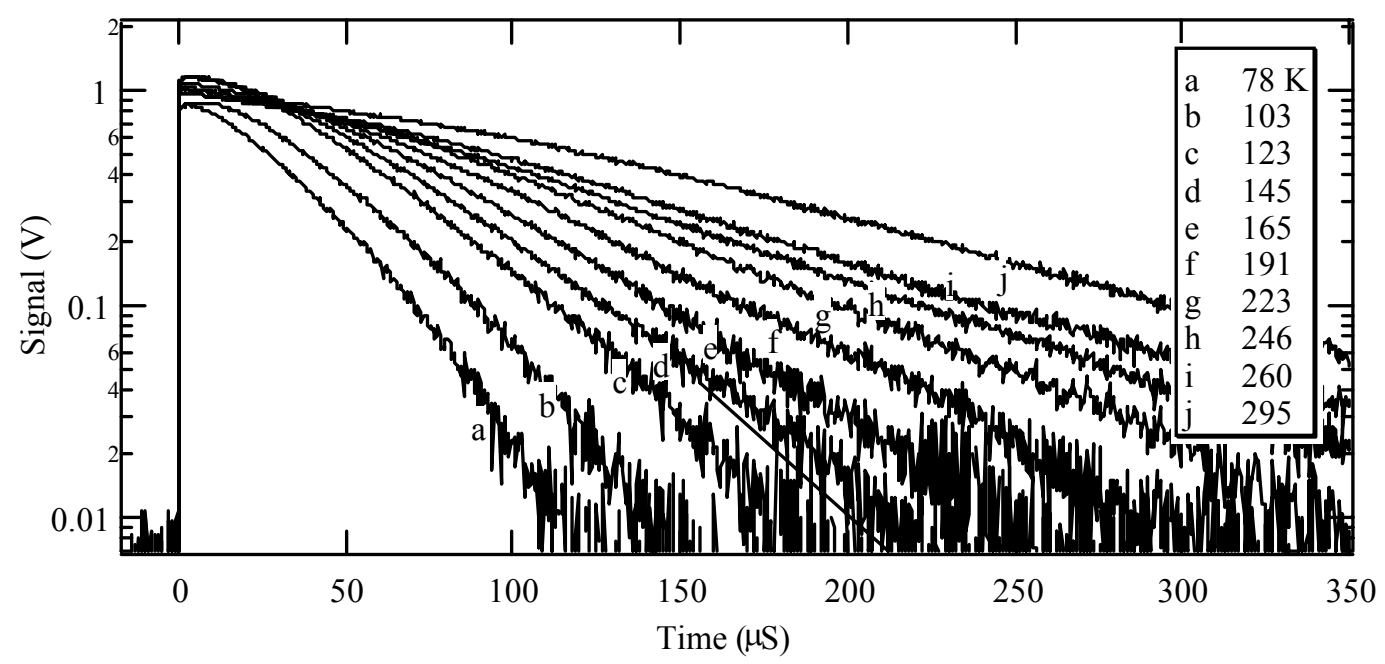

FIGURE 1. Excess-carrier-decay experimental data of the Si sample for various temperatures.

$$
\frac{\mathrm{d} \rho}{\mathrm{dt}}=-\frac{\left(\mathrm{n}_{\mathrm{o}}+\rho\right)\left(\mathrm{p}_{\mathrm{o}}+\rho\right)-\mathrm{n}_{\mathrm{i}}^{2}}{\frac{1}{\sigma_{\mathrm{p}} \mathrm{v}_{\mathrm{th}} \mathrm{N}_{\mathrm{T}}}\left(\mathrm{n}_{\mathrm{o}}+\rho+\mathrm{n}_{1}\right)+\frac{1}{\sigma_{\mathrm{n}} \mathrm{v}_{\mathrm{th}} \mathrm{N}_{\mathrm{T}}}\left(\mathrm{p}_{\mathrm{o}}+\rho+\mathrm{p}_{1}\right)}
$$

where $\rho$ is excess-carrier density, $n_{o}$ and $p_{o}$ are equilibrium electron and hole densities, $n_{i}$ is intrinsic density, $\sigma_{\mathrm{n}}$ and $\sigma_{\mathrm{p}}$ are electron and hole capture cross sections, $\mathrm{v}_{\mathrm{th}}$ is thermal velocity, $\mathrm{N}_{\mathrm{T}}$ is recombination center density, and $\mathrm{n}_{1}$ and $\mathrm{p}_{1}$ are SRH-detailed balance terms. The number of defects and value of cross sections are unknown, but their product corresponds with doping density and excess-carrier injection-level density.

A computer model incorporating SRH recombination of Eq. 1 calculates decay curves shown in Fig. 2. The majority-electron capture cross section, $\sigma_{\mathrm{n}}$, is roughly four

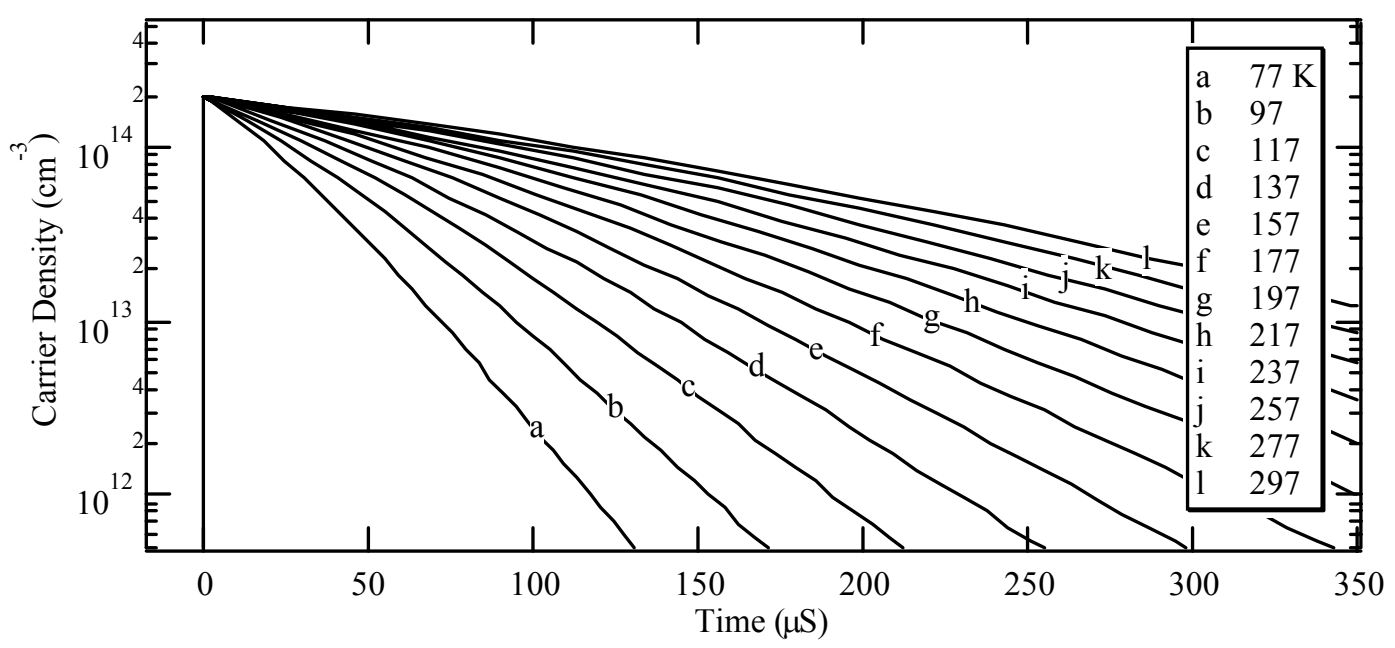

FIGURE 2. Modeling of Si sample excess-carrier decay using SRH recombination. 
times larger than the minority-hole capture cross section, $\sigma_{\mathrm{p}}$. These parameters are similar to injection-level spectroscopy data reported on similar $\mathrm{Si}$ samples (20). The temperature dependence for the capture cross sections is modeled to vary as $\mathrm{T}^{-2}$, corresponding to a Coulomb-attractive recombination center.

The GaAs sample's measured excess-carrier-decay curves are plotted in Fig. 3.

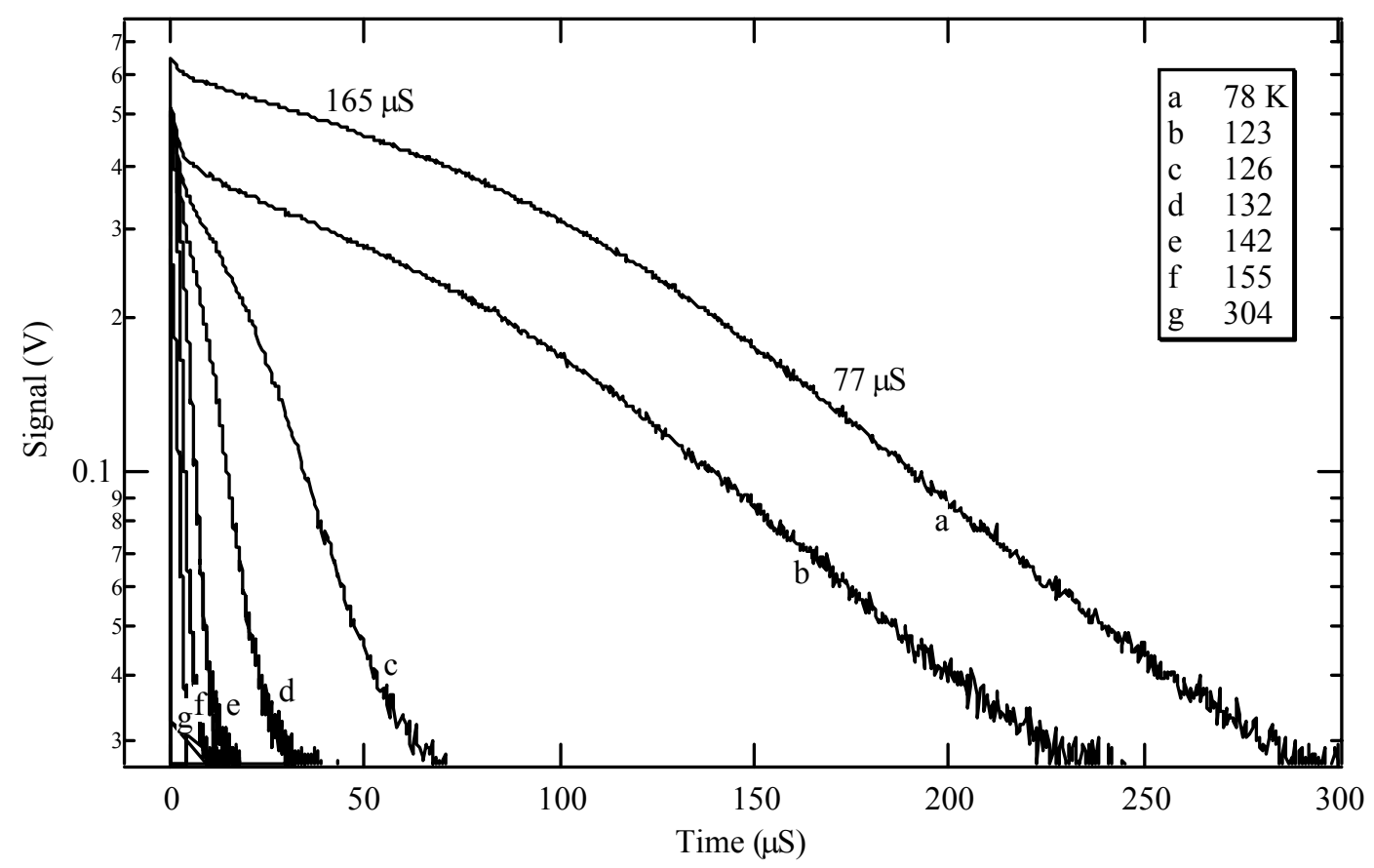

FIGURE 3. Excess-carrier-decay experimental data of the GaAs sample for various temperatures.

A high- and low-injection level can be seen similar to the Si sample. However, unlike the Si case, the GaAs lifetimes decrease rapidly with increasing temperature. The mid- to lowinjection regions are similarly modeled for GaAs as in the previous Si sample by using a midgap SRH recombination center. Also, there is an initial fast decay rate at the highest excesscarrier concentration. To account for this highest injection-level decay, radiative recombination is added to the model of Eq. 1. For GaAs, this factor reduces the excess carriers according to Eqs. 2 and 3 (21).

$$
\begin{gathered}
\frac{d \rho}{d t}=-B\left(n_{o}+\rho\right)\left(p_{o}+\rho\right) \\
B=3 \times 10^{-10}\left(\frac{E_{g}}{1.5}\right)^{2}\left(\frac{300}{T}\right)^{1.5} \mathrm{~cm}^{3} / \mathrm{s}
\end{gathered}
$$

For lifetime to decrease with increasing temperature, the capture cross sections must increase, which may be described by a Coulomb-repulsive model. However, both the highand low-injection lifetimes decrease, and a recombination center cannot be Coulomb repulsive for both electrons and holes. Instead, this increased lifetime is modeled by adding a shallow trapping center below the conduction band. When an electron is captured in this trapping 
center, it is more likely to be re-emitted to the conduction band rather than the center capturing a hole for recombination. When traps are present, the experimentally observed photoconductive-decay time can be longer than the carrier lifetime (22). If the density of trapped carriers is comparable to or larger than the density of free carriers, then the thermal emission of the trapped carriers during the course of decay can prolong the decay time (22). At near-liquid nitrogen temperatures, the Fermi level lies closer to the conduction band and above the trap's energy level. Thus, the traps are mostly occupied at low temperature, making their density comparable or even larger than the density of electrons in the conduction band. Then, as temperature increases and the Fermi level moves further below the conduction band, it passes below the trap level. The traps empty, and they less effectively prolong the decay. The modeled results are graphed in Fig. 4.

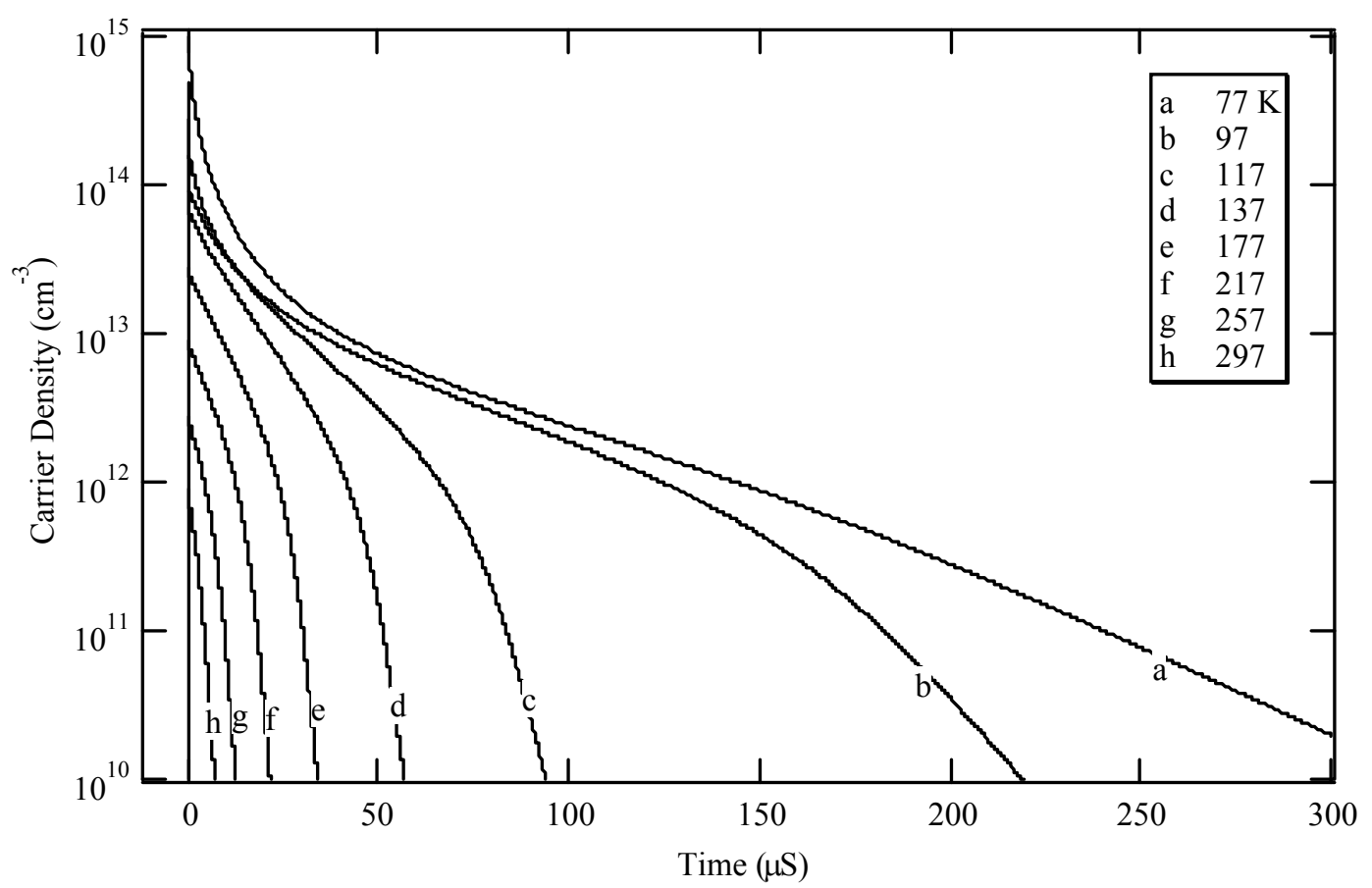

FIGURE 4. Modeling of GaAs sample excess-carrier-density decay.

For the above results, a trapping level is introduced at $70 \mathrm{meV}$ below the conduction band with a density of $10^{14} \mathrm{~cm}^{-3}$. The minority-carrier capture cross section is six times larger than that for the majority carrier. The product of their values with the number of SRH recombination centers determines the lifetimes as mid- and low-injection. The residual n-type doping density is mid-10 12 where there is a transition from SRH high- to low-injection regimes. The sharp increase in decay rate versus temperature corresponds to the Fermi level passing through the trap energy level.

\section{CONCLUSIONS}

A contactless, UHFPCD lifetime measurement apparatus gives the capability to measure temperature-dependent minority-carrier lifetime for a wide range of materials including, but not limited to, Si and GaAs. Other materials such as InGaAs, InP, CdS, and HgCdTe have 
also been measured. Such a technique can quickly and easily provide additional defect characterization for a variety of semiconductor materials without requiring additional processing steps for contacts or device construction.

\section{ACKNOWLEDGMENTS}

The authors would like to thank both UniSil and Spire for providing the Si and GaAs samples, respectively.

\section{REFERENCES}

1. Schroder, D. K., IEEE Trans. Electron Devices 44, 160-170 (1997).

2. Horanyi, T. S., Tutto, P., and Kovacsics, C., J. Electrochem. Soc. 143, 216-220 (1996).

3. Anttila, O. J., and Tilli, M. V., J. Electrochem. Soc. 139, 1751-1756 (1992).

4. $\quad$ Ferenczi, G., Pavelka, T., and Tutto, P., Jap. J. of Appl. Phys. 30, 3630-3633 (1991).

5. $\quad$ Ling, C. H., and Cheng, Z. Y., Appl. Phys. Lett. 71, 3218-3220 (1997).

6. Daio, H., Yakushiji, K., Buczkowski, A., and Shimura, F., Materials Science Forum, Switzerland: Trans Tech Publications, 1995, 196-201, pp. 1817-1822.

7. Daio, H., Buczkowski, A., and Shimura, F., J. Electrochem. Soc. 141, 1590-1593 (1994).

8. Hayamizu, Y., Hamaguchi, T., Ushio, S., Abe, T., and Shimura, F., J. Appl. Phys. 69, 3077-3081 (1991).

9. $\quad$ Shimura, F., Okui, T., and Kusama, T., J. Appl. Phys. 67, 7168-7171 (1990).

10. Kirino, Y., Buczkowski, A., Radzimski, Z. J., Rozgonyi, G. A., and Shimura, F., Appl. Phys. Lett. 57, 2832-2834 (1990).

11. Ahrenkiel, R. K., AIP Conference Proceedings, AIP Press 353, 1996, pp. 161-174.

12. Yablonovitch E., and Gmitter, T. J., Solid-State Electronics 35, 261-267 (1992).

13. Hwang, S. H., Eo, Y. P., Seo, J. H., Whang, K. W., Yoon, E., and Tae, H. S., J. Vac. Sci. Technol. A 14, 1033-1036 (1996).

14. Maekawa, T., Yamagishi, Y., and Inoue, S., Jpn. J. Appl. Phys. 32, 5740-5747 (1993).

15. Nicollian, E. H., and Brews, J. R., MOS (Metal Oxide Semiconductor) Physics and Technology, New York: John Wiley \& Sons, 1982, p. 645.

16. Rose, A., Concepts in Photoconductivity and Allied Problems, Huntington, NY: Robert E. Krieger Publishing Co., 1978, ch. 7, pp. 121-128.

17. Lax, M., Physical Review 119, 1502-1523 (1960).

18. Shockley, W., and Read, W. T., Jr., Physical Review 87, 835-842 (1952).

19. Hall, R. N., Physical Review 87, 387 (1952).

20. Ahrenkiel, R. K., and Johnston, S., Mater. Res. Soc. Proc., Pittsburgh, PA, 1998 (in press).

21. Ahrenkiel, R. K., Semiconductors and Semimetals 39, New York: Academic Press, 1993, ch. 2, pp. 57-65.

22. Bube, R. H., Photoconductivity of Solids, New York: John Wiley \& Sons, Inc., 1960, ch. 3, pp. 5674. 